\title{
Physiological Traits of Durum Wheat (Triticum durum Desf.) and Bread Wheat (Triticum aestivum L.) Genotypes under Drought Stress
}

\author{
Tofig I. Allahverdiyev \\ Plant Physiology and Biotechnology Department, Research Institute of Crop Husbandry, Baku, Azerbaijan \\ Email: tofig_1968@mail.ru
}

Received 13 July 2015; accepted 22 August 2015; published 25 August 2015

Copyright (c) 2015 by author and Scientific Research Publishing Inc. This work is licensed under the Creative Commons Attribution International License (CC BY). http://creativecommons.org/licenses/by/4.0/

\begin{abstract}
We aimed to study impact of drought stress on physiological traits of field grown 8 durum and 14 bread wheat genotypes. Drought caused decrease of leaf gas exchange parameters-photosynthesis rate $\left(P_{n}\right)$, stomatal conductance $\left(g_{s}\right)$, and transpiration rate $(E)$, an increase of intercellular $\mathrm{CO}_{2}$ concentration $\left(\mathrm{C}_{\mathrm{i}}\right)$. Area (LA) and dry mass of leaves per stem, leaf area index (LAI) of genotypes significantly reduced from booting to watery ripe stages. Water deficiency led to a decrease of chlorophyll $a, b(C h l a, b)$ and carotenoids $(\operatorname{Car}(x+c)$ content, relative water content $(\mathrm{RWC})$. Water stress more affected on LA than leaf dry mass of wheat genotypes, leaf specific mass (LSM) increased. The Chl $(a+b)$ content, $P_{n}$ and yield of bread wheat genotypes were relatively higher than durum wheat ones. Physiological traits may be reliable for selection of drought tolerant wheat genotypes.
\end{abstract}

Keywords

Wheat, Photosynthesis Rate, Stomatal Conductance, Chlorophyll, Water Stress

\section{Introduction}

Plants are often subjected to periods of unfavorable conditions (drought, high light and temperature) during their life cycle in the field. Drought is the most severe stress and the main cause of significant losses in growth, productivity of crop plants, and finally their yields [1]. Changes in global climate are forecasted to increase the extension of drought-prone areas [2]. The problem of drought is acute in developing countries of the world, where 
about $37 \%$ of wheat growing areas are semi-arid in which available limited soil moisture constitutes a primary hurdle in way of wheat production [3]. Drought is a serious problem for wheat production in rain-fed regions of Azerbaijan. Wheat is a staple food in human nutrition, and is a source of energy from carbohydrates and proteins. Water stress induces many physiological, biochemical and molecular responses on plants; so that plants are able to develop tolerance mechanisms which will provide to be adapted to limited environmental conditions [4]. A decline in photosynthesis in water stressed plants can be caused by stomatal closure and impairments in photochemical and/or biochemical reactions [5]. In the field water stress strengthening during post-anthesis stages is also accompanied by high light intensity and temperature. Total plant carbon uptake is further reduced due to the concomitant or even earlier inhibition of growth [6]. Reduction in Chl a and b has been reported in some earlier studies on different crops, e.g., sunflower Heliantus annuus [7], wheat, Triticum aestivum [8]. The decrease may be attributed to accelerated breakdown of Chl rather than its slow synthesis [9].

For identifying of drought tolerance of wheat, we need to test a number of genotypes in the field. It is revealed that varieties, with higher leaf turgor and relative water content under stress conditions, are more drought tolerant and give higher yield than others [10] [11]. We aimed to study the effect of soil water deficit on leaf gas exchange parameters, photosynthetic pigments content, RWC, LA, dry mass, LAI of durum wheat (Triticum durum Desf.) and bread wheat (Triticum aestivum L.) genotypes.

\section{Material and Methods}

\subsection{Plant Materials and Growth Conditions}

Eight durum wheat genotypes (Garagylchyg 2,Vugar, Shiraslan 23, Barakatli 95, Alinja 84, Tartar, Sharg, Gyrmyzybugda), fourteen bread wheat genotypes (Nurlu 99, Gobustan, Akinchi 84, Giymatli 2/17, Gyrmyzy gul1, Azamatli 95, Tale 38, Ruzi 84, Pirshahin1, $12^{\text {nd }}$ FAWWON№97, $4^{\text {th }}$ FEFWSN№50, Gunashli, Dagdash, Saratovskaya29) were employed in this study. Wheat genotypes were grown in the field of Plant Physiology and Biotechnology Department of Research Institute of Crop Husbandry, Azerbaijan, during the growing season of 2013-2014. Sowing was down 25-26 October, at an average density 400 seeds $\cdot \mathrm{m}^{-2}$ with self-propelled mechanical planter in $1 \mathrm{~m} \times 10 \mathrm{~m}$ plots, consisting of 7 rows placed $15 \mathrm{~cm}$ apart. Each genotype was sown with three replications both under irrigated and rain-fed conditions. Irrigated plots were watered with the appearance of seedlings, at stem elongation, and grain filling stages. Rain-fed plots were not watered during ontogeny. Soil moisture content (\% of the field capacity) was determined in the 0 - 20, $20-40,40-60 \mathrm{~cm}$ depths, and was about $60.4 \%$ under irrigated, $33 \%$ under rain-fed conditions during booting stage, $60 \%$ under irrigated, $28 \%$ under rain-fed conditions during watery ripe stage.

\subsection{Measurements}

Gas exchange parameters were measured with a Portable Photosynthesis System LI-6400XT (LI-COR Biosciences, Lincoln, NE, USA), equipped with $6 \mathrm{~cm}^{2}$ leaf chamber. Light intensity measured by using Light-Meter LI-250A (LI-COR Biosciences) equipped with Pyranometer PY 71968 (LI-COR Biosciences). A lux meter data (klux) is converted into photosynthetically active radiation (PAR) unit ( $\mu \mathrm{mol}$ photons $\mathrm{m}^{-2} \cdot \mathrm{s}^{-1}$ ) multiplying by 1000 and then divided into 54. Gas exchange measurements done during booting stage (Feekes Stage 10) and watery ripe stage (Feekes Stage 10.5.4). Measurements carried out between 10:00 and 12:00 a.m. Data logging started after 45 seconds of insertion the leaf into chamber. Five-seven replicate measurements were conducted for each genotype. As Table 1 shows the maximum $P_{n}$ and PAR is observed to 13:00 a.m. of the day. Pigments content and RWC measurements done during kernels watery ripe stage. Leaf Chl a, b and Car $(x+c)$ content determined following the method of Lichtenthaler (1987) [12] with little modification. About $0.1 \mathrm{~g}$ fresh leaves were ground in $96 \%$ ethanol for the extraction of chlorophyll and carotenoids. Absorbance of the supernatant was recorded at 664, 648 and $470 \mathrm{~nm}$ spectrophotometrically (Genesys 20, Thermo Scientific, USA). Pigments content calculated by the following formulas.

$$
\begin{aligned}
& \text { Chla }=\left(13.36 \cdot A_{664}-5.19 \cdot A_{648}\right) \cdot 25 / D W ; C h l b=\left(27.43 \cdot A_{648}-8,12 \cdot A_{664}\right) \cdot 25 / D W \\
& \operatorname{Car}(x+c)=\left(4.785 \cdot A_{470}+3.657 \cdot A_{664}-12.76 \cdot A_{648}\right) \cdot 25 / D W
\end{aligned}
$$

LA measured with an area meter (AAC-400, Hayashi Denkon Co, LTD, Japan). Data corresponds to the LA 
Table 1. Daily variation of $\mathrm{P}_{\mathrm{n}}$ in dependence on PAR. (Data are mean \pm SE from six replication).

\begin{tabular}{|c|c|c|c|c|c|c|c|}
\hline & 09:00 a.m. & 11:00 a.m. & 13:00 a.m. & 15:00 a.m. & 17:00 a.m. & 18:00 a.m. & 19:00 a.m. \\
\hline PAR, $\underset{\mathrm{m}^{-2} \cdot \mathrm{s}^{-1}}{\boldsymbol{\mu} \text { mol photons }}$ & 1003 & 1520 & 1706 & 1561 & 1391 & 895 & 667 \\
\hline$P_{\mathrm{n}}, \mu \mathrm{mol} \mathrm{CO} \mathrm{C}_{2} \mathrm{~m}^{-2} \cdot \mathrm{s}^{-1}$ & $10.68 \pm 1.4$ & $13.55 \pm 0.36$ & $17.9 \pm 0.94$ & $16.98 \pm 1.23$ & $11.56 \pm 0.55$ & $5.95 \pm 0.62$ & $3.79 \pm 0.32$ \\
\hline
\end{tabular}

per stem. The flag leaf RWC determined gravimetrically. Immediately after cutting leaves were preserved within plastic bags and in time transferred to the laboratory to determine fresh weight (FW). Turgid weight (TW) was determined after saturating leaves in distilled water for $24 \mathrm{~h}$ at room temperature in dark place. After saturating, leaves were carefully blotted dried with tissue paper. Dry weight (DW) was measured after oven drying the leaves samples at $105^{\circ} \mathrm{C}$ for $24 \mathrm{~h}$. RWC calculated by using the following formula:

$\mathrm{RWC}(\%)=(\mathrm{FW}-\mathrm{DW}) /(\mathrm{TW}-\mathrm{DW}) \times 100$. Mean and standard errors of dates calculated by Excel program.

\section{Results}

\subsection{Effect of Drought Stress on Gas Exchange Parameters}

High $P_{n}$ is considered to be one the most important breeding strategies for crop improvement [13] and greater success might be expected for higher rates under water stress [2]. Soil water deficit caused reduction of $P_{n}$ (Figure 1). The $P_{n}$ in the booting stage was higher than in the watery ripe stage. A higher $P_{n}$ during booting stage was observed in flag leaf of bread wheat genotypes under irrigated condition. Relatively high $\mathrm{P}_{\mathrm{n}}$ under irrigated condition during watery ripe stage retained in genotypes Tartar, Giymatli2 $\backslash 17$, Tale 38, Pirshain 1 . Reduction in $P_{n}$ under drought stress was stronger during watery ripe stage. A strong reduction of $P_{n}$ under water stress in both stages was observed in genotypes Barakatli 95, Nurlu 99, Gobustan, Akinchi 84. Low decrease in $\mathrm{P}_{\mathrm{n}}$ during both stages observed in genotypes Garagylchyg 2, Vugar, Shiraslan 23, Gyrmyzybugda, Gyrmyzy gul 1, Pirshahin 1 and $4^{\text {th }}$ FEFWSN№50.

Water deficit led to a decrease in $g_{s}$ (Figure 2). The $g_{s}$ was higher during booting than watery ripe in most genotypes both under irrigated and rain-fed conditions with exceptions Sharg, Gyrmyzybugda, Dagdash and Saratovskaya 29. Strong decrease of $g_{s}$ in both stages was found in genotypes Tartar, Nurlu 99, Gobustan, Akinchi 84, Ruzi 84. In some genotypes (Shiraslan 23, Sharg, Giymatli 2/17, Gyrmyzy gul 1, Tale 38, Saratovskaya 29) stronger reduction was expressed in the stage of watery ripe.

The lowest decrease in $g_{s}$ during both stages observed in genotypes Shiraslan 23, Sharg, Gyrmyzybugda, Gyrmyzy gul 1, $12^{\text {nd }}$ FAWWON№97, Gunashli, $4^{\text {th }}$ FEFWSN№50 and Dagdash. We observed an increase of $g_{s}$ in genotypes Gyrmyzybugda, Dagdash, Saratovskaya 29 during watery ripe stage.

We found an increase in $C_{i}$ under drought conditions which strongly expressed in the stage of watery ripe (Figure 3). Significant increase in $C_{i}$ during booting stage was found in genotypes Shiraslan 23, Sharg, Nurlu 99, $4^{\text {th }}$ FEFWSN№50, Saratovskaya 29 and in genotypes Barakatli 95, Alinja 84, Tartar, Tale 38, Ruz 84, Gunashli during watery ripe stage.

Water stress led to decrease in E (Figure 4). The E was higher during watery ripe stage in most genotypes. The E was relatively higher in genotypes of bread wheat than durum wheat ones. Strong reductions in E was detected in genotypes Garagylchyg 2, Vugar, Barakatli 95, Nurlu 99, Gobustan, Akinchi 84, Gyrmyzy gul 1, Azamatli 95, Ruzi 84, Gunashli and $4^{\text {th }}$ FEFWSN№50 during booting stage. Reductions in E were higher in genotypes Tartar, Akinchi 84, Tale 38, Ruzi 84, Dagdash and Saratovskaya 29 during watery ripe stage. Higher decrease of $\mathrm{E}$ during both stages detected in genotypes Garagylchyg 2, Akinchi 84, Gyrmyzy gul 1, Ruzi 84. Low decrease in E during both stages was observed in genotypes Shiraslan 23, Barakatli 95, Alinja 84, Sharg, Gyrmyzybugda, Giymatli 2/17, Pirshahin 1, and $12^{\text {nd }}$ FAWWON№97.

\subsection{Effect of Water Stress on Leaf RWC}

The RWC is an important indicator of the state of water balance of a plant. The RWC of flag leaf of most genotypes was greater than $80 \%$ during post anthesis grain formation stage under irrigated condition (Figure 5). Reductions in RWC of genotypes Shiraslan 23, Akinchi 84, Tale 38, $12^{\text {nd }}$ FAWWON97, Saratovskaya 29 was nonsignificant. Comparatively low RWC was found in genotypes Garagylchyg 2, Shiraslan 23, Nurlu 99, Akinchi 84. Significant reduction was detected in genotypes Barakatli 95, Tartar, Nurlu 99, Gyrmyzy gul 1, Gunashli. 


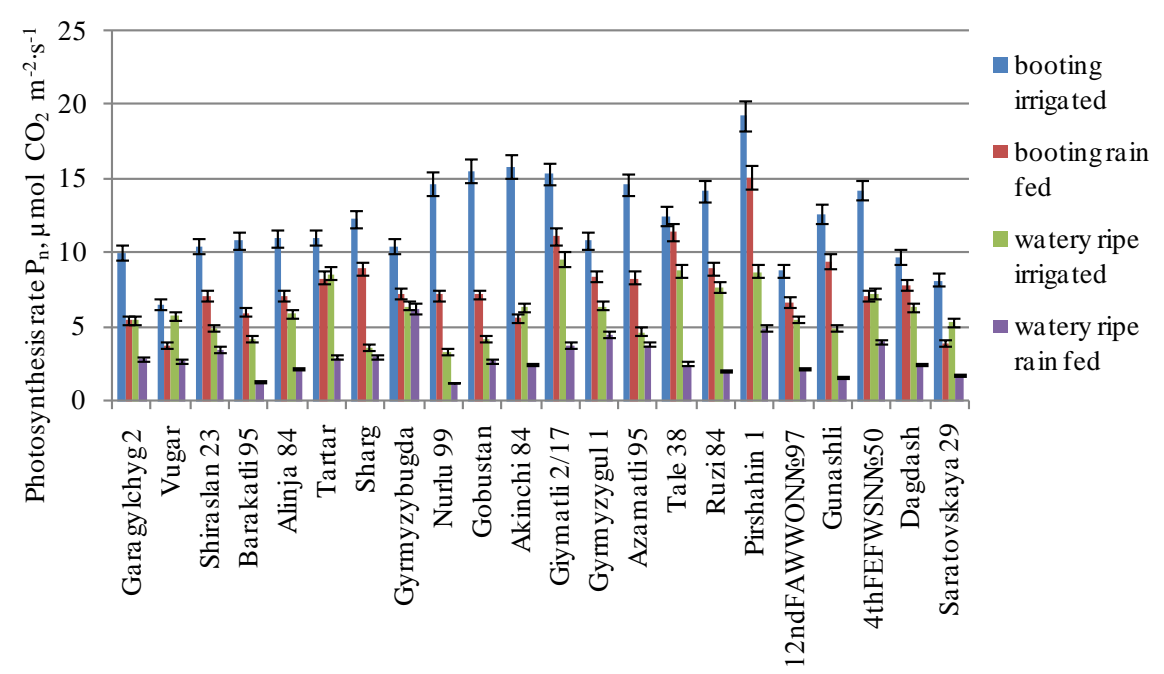

Figure 1. Effect of drought stress on $\mathrm{P}_{\mathrm{n}}$. Data are mean $\pm \mathrm{SE}$ from seven replication.

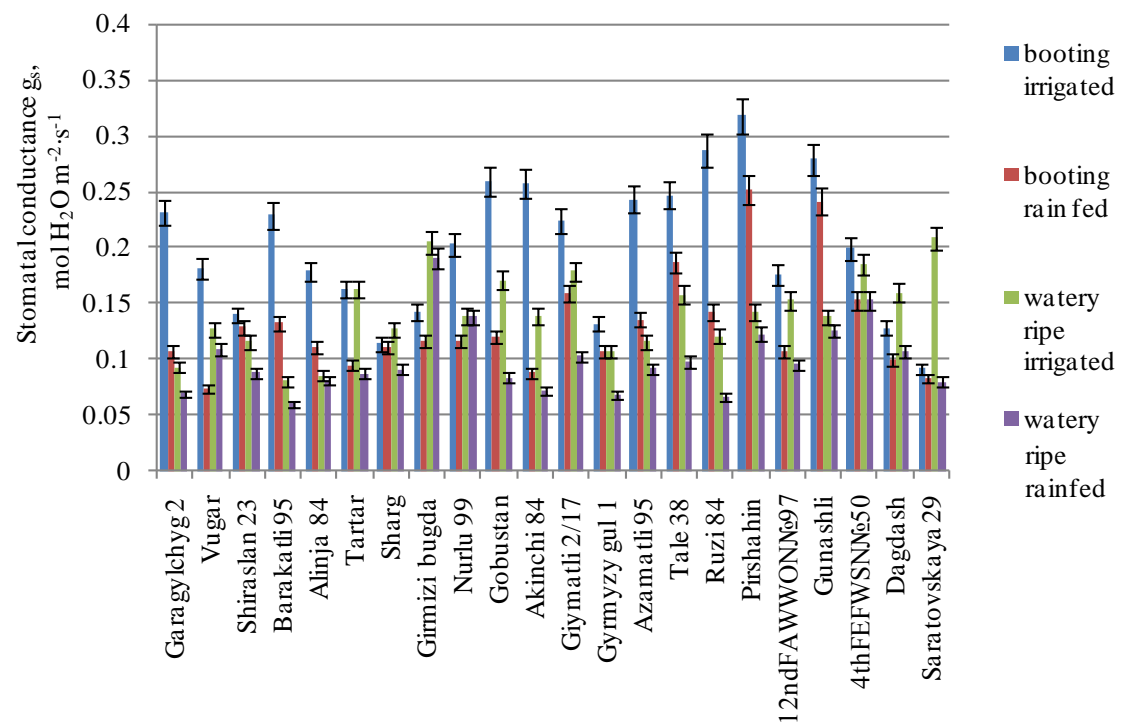

Figure 2. Effect of drought stress on $g_{s}$. Data are mean \pm SE from seven replication.

We observed an increase in RWC of genotype Sharg. The RWC of genotypes Vugar, Tartar, Gyrmyzybugda, Giymatli 2/17, Azamatli 95, Tale 38, Ruzi 84, Pirshahin1, $12^{\text {nd }}$ FAWWON97, $4{ }^{\text {th }}$ FEFWSN50, Dagdash, Saratovskaya 29 retained at relatively high level under rain-fed conditions. This may be due to accumulation of osmotic active compouds, regulation of water loss or deep root system.

\subsection{Effect of Water Stress on Chl $(\mathrm{a}+\mathrm{b})$ and Car $(\mathrm{x}+\mathrm{c})$ Content}

Drought caused reduction of $\mathrm{Chl}(\mathrm{a}+\mathrm{b})$ and $\mathrm{Car}(\mathrm{x}+\mathrm{c})$ contents. The Chl $(\mathrm{a}+\mathrm{b})$ content was greater in the flag leaf of bread wheat genotypes than durum wheat ones (Table 2). Higher Chl $(\mathrm{a}+\mathrm{b})$ content was detected in durum wheat genotypes Barakatli 95, Tartar, bread wheat genotypes Gobustan, Akinchi 84, Gyrmyzy gul 1, Tale 38, $4^{\text {th }}$ FEFWSNNNo50, Dagdash, Saratovskaya 29 under irrigated condition. Drought led to a decrease of Chla/b ratio. But in some genotypes we found no changes or an increase of $\mathrm{Chl} a / b$ ratio. The $\mathrm{Chl}(\mathrm{a}+\mathrm{b}) / \mathrm{Car}(\mathrm{x}+\mathrm{c})$ ratio was decreased under water stress. We observed an increase of $\mathrm{Chl}(\mathrm{a}+\mathrm{b}) / \mathrm{Car}(\mathrm{x}+\mathrm{c})$ ratio in genotypes Garagylchyg 2 and Alinja 84. Strong reduction of Chl $(\mathrm{a}+\mathrm{b})$ content was detected in genotypes Garagylchyg 2, Tartar, Nurlu 99, Gobustan, Akinchi 84 and Gunashli. Less reduction in Chl $(\mathrm{a}+\mathrm{b})$ content was detected in genotypes Vugar, Sharg, Gyrmyzybugda, Azamatli 95, $12^{\text {nd }}$ FAWWON97, Dagdash and Saratovskaya 29. Water deficit had more 


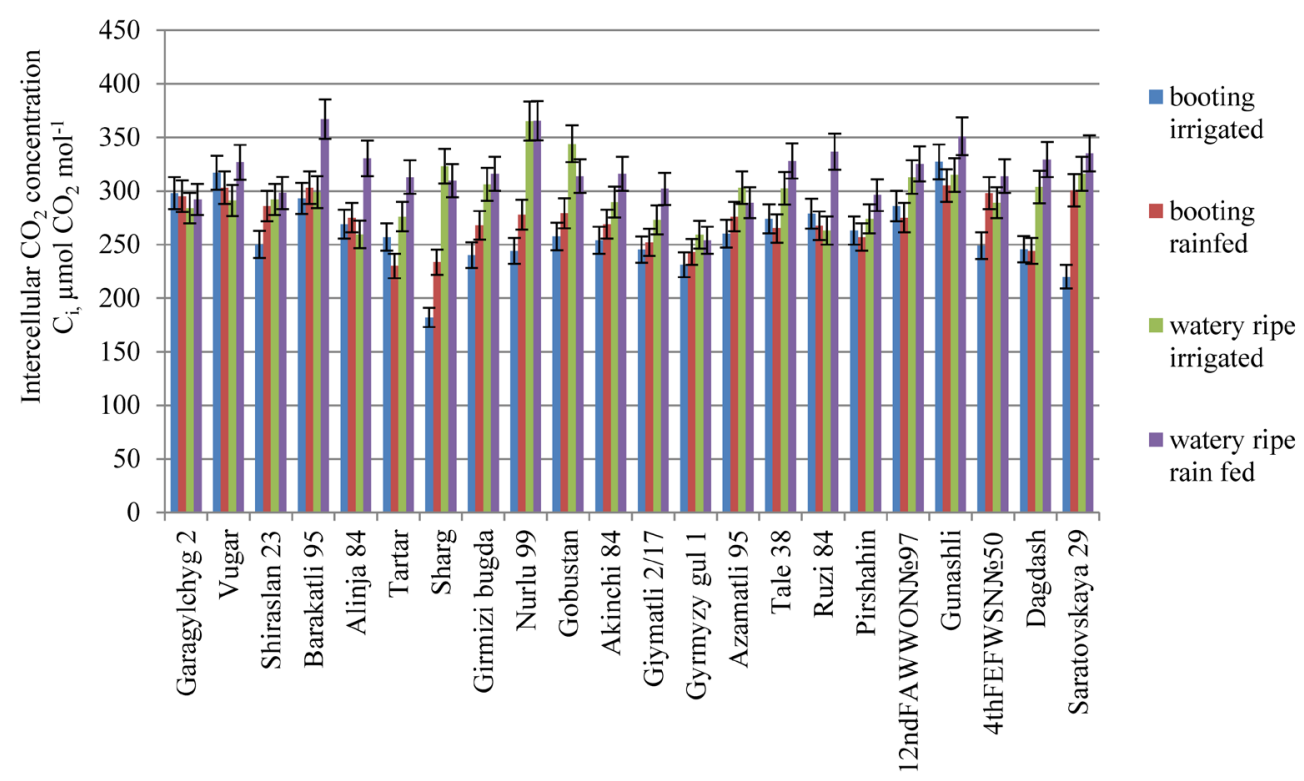

Figure 3. Effect of drought stress on $\mathrm{C}_{\mathrm{i}}$. Data are mean $\pm \mathrm{SE}$ from seven replication.

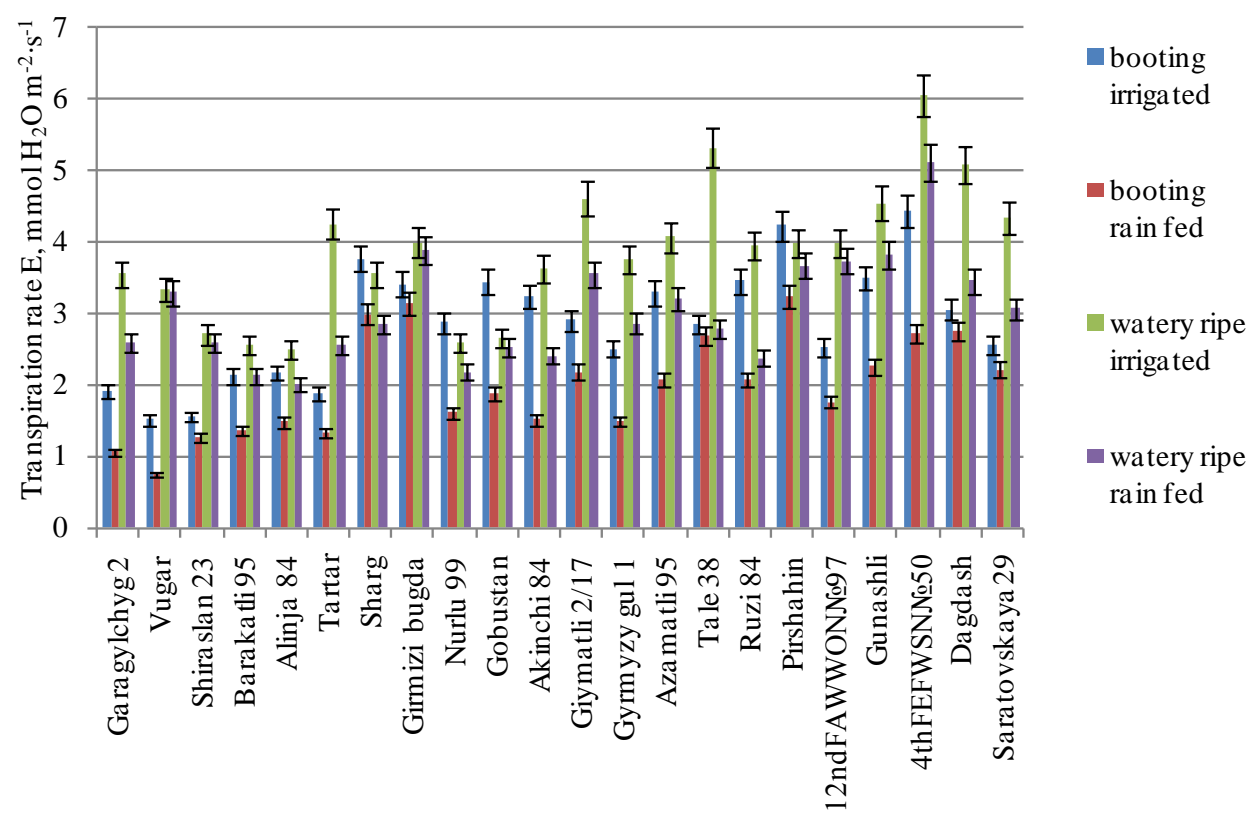

Figure 4. Effect of water stress on E. Data are mean \pm SE from seven replication.

influence on Chl $(\mathrm{a}+\mathrm{b})$ content than the content of $\mathrm{Car}(\mathrm{x}+\mathrm{c})$.

\subsection{Effect of Water Stress on LA and Leaf Dry Mass Per Stem}

Assimilation area of leaves were greater in genotypes of durum wheat than in genotypes of bread wheat ones (Figure 6). Bread wheat genotypes Gobustan, Akinchi 84, Giymatli 2\17, $4^{\text {th }}$ FEFWSN№50 formed larger leaf area. Strong reduction of leaf area to watery ripe stage was observed in durum wheat genotypes Garagylchyg 2, Barakatli 95, Tartar and almost in all genotypes of bread wheat under drought stress. Reduction of LA under drought stress was relatively small in genotypes Vugar, Shiraslan 23, Sharg, Gyrmyzy bugda, Giymatli $2 \backslash 17$ and Dagdash. Reduction in LA by water stress is an important cause of reduced crop yield through decline in photosynthesis [14]. 


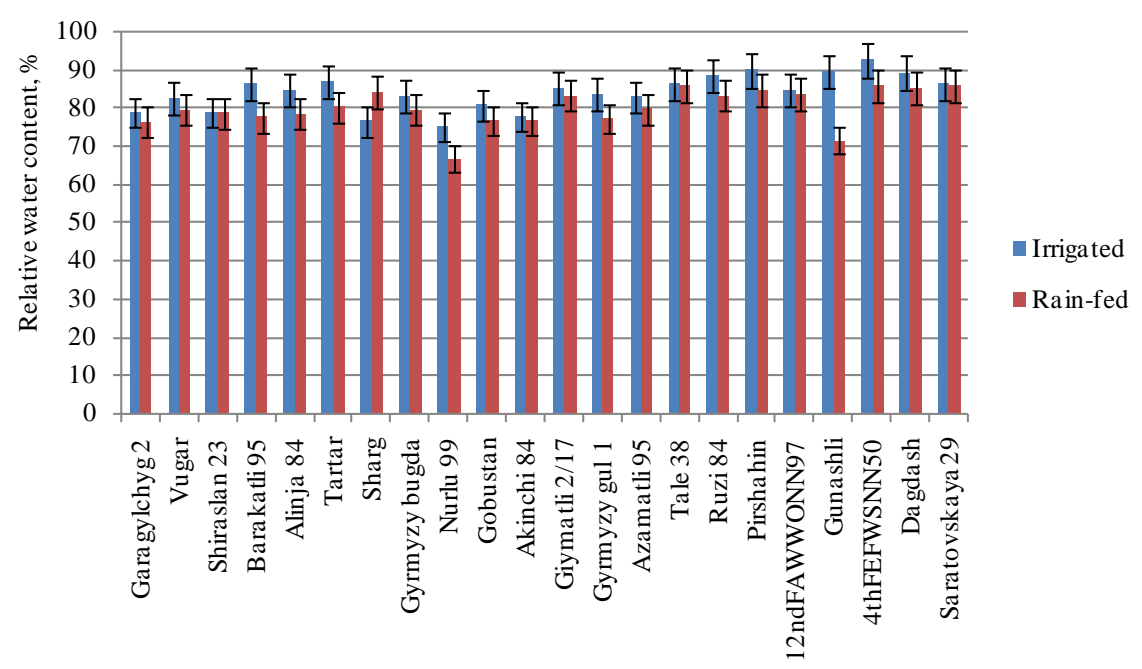

Figure 5. Effect of water stress on RWC. Data are mean \pm SE from three replication.

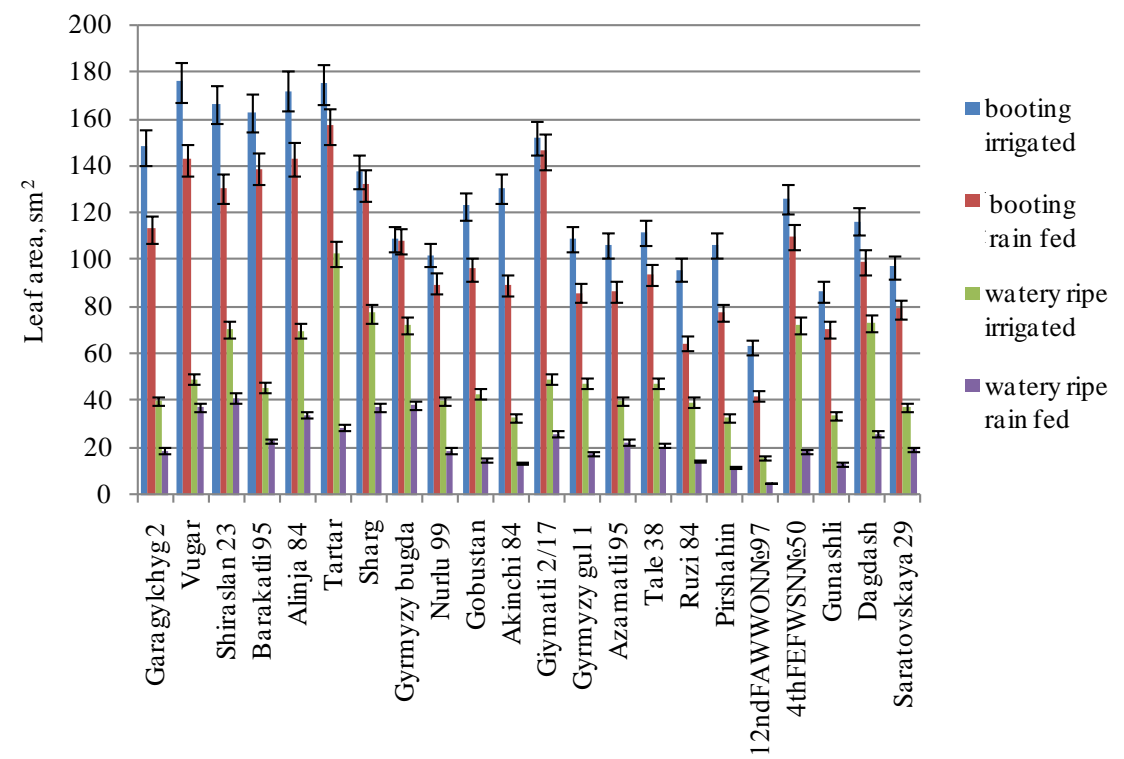

Figure 6. Effect of water stress on leaf area per stem. Data are mean \pm SE from five replications.

As expected large LA of durum wheat genotypes accumulated higher dry matter (Figure 7). Reduction in dry matter under water deficiency was not strong during booting stage. However strong decrease in dry matter of leaves to watery ripe stage was detected in genotypes Garagylchyg 2, Barakatli 95, Tartar, Nurlu, Gobustan, Akinchi 84, $12{ }^{\text {nd }}$ FAWWON№97 and Gunashli.

\subsection{Effect of Water Stress on LAI}

LAI was higher in genotypes Garagylchyg 2, Vugar, Shiraslan 23, Barakatli 95, Giymatli 2\17, Gyrmyzy gul 1, Tale 38, $4^{\text {th }}$ FEFWSN№50 under irrigated condition during booting stage (Figure 8). This trait remain relatively higher to watery ripe stage in genotypes Tartar, Gyrmyzybugda, Gyrmyzy gul 1, $4{ }^{\text {th }}$ FEFWSN№50 and Dagdash under irrigated condition. Water stress strengthened leaf senescence. Strong reduction of LAI under water stress was observed in genotypes Garagylchyg 2, Vugar, Barakatli 95, Akinchi 84, Ruzi 84, Pirshahin 1 and $12^{\text {nd }}$ FAWWON№97, less reduction in genotypes Shiraslan 23, Gyrmyzybugda, Gunashli, Saratovskaya 29. Reduction of LAI during watery ripe stage was deep, more pronounced in genotypes Barakatli 95, Tartar, Akinchi 84, Ruzi 84, Pirshahin 1, $12^{\text {nd }}$ FAWWON№97, $4^{\text {th }}$ FEFWSN№50 and Dagdash. 
Table 2. Effect of water stress on Chl a, b and carotenoids content Note: I-irrigated: R-f-rain-fed.

\begin{tabular}{|c|c|c|c|c|c|c|c|}
\hline \multicolumn{2}{|c|}{ Wheat genotypes } & $\underset{\mathrm{mg} \cdot \mathrm{g}^{-1} \mathrm{dw}}{\mathrm{Chl} \mathrm{a}}$ & $\begin{array}{c}\text { Chl b } \\
\mathrm{mg} \cdot \mathrm{g}^{-1} \mathrm{dw}\end{array}$ & 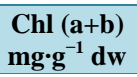 & $\begin{array}{l}\text { Car }(x+c) \\
\mathrm{mg} \cdot \mathrm{g}^{-1} \mathrm{dw}\end{array}$ & Chl a/b & $\begin{array}{l}\text { Chl }(a+b) / \\
\text { Car }(x+c)\end{array}$ \\
\hline \multicolumn{8}{|c|}{ Triticum durum Desf. } \\
\hline \multirow{2}{*}{ Garagylchyg 2} & I & 6.34 & 2.56 & 8.9 & 1.48 & 2.47 & 6.01 \\
\hline & R-f & 3.08 & 1.75 & 4.83 & 0.63 & 1.77 & 7.66 \\
\hline \multirow{2}{*}{ Vugar } & I & 6.29 & 2.70 & 8.98 & 1.33 & 2.33 & 6.75 \\
\hline & $R-f$ & 6.04 & 2.70 & 8.74 & 1.40 & 2.23 & 6.24 \\
\hline \multirow{2}{*}{ Shiraslan23 } & I & 5.54 & 2.82 & 8.36 & 1.02 & 1.96 & 8.23 \\
\hline & $R-f$ & 4.16 & 2.15 & 6.31 & 1.01 & 1.93 & 6.27 \\
\hline \multirow{2}{*}{ Barakatli95 } & I & 6.85 & 2.91 & 9.75 & 1.53 & 2.36 & 6.37 \\
\hline & $R-f$ & 5.36 & 2.40 & 7.76 & 1.15 & 2.23 & 6.75 \\
\hline \multirow{2}{*}{ Alinja84 } & $\mathrm{I}$ & 5.68 & 2.67 & 8.36 & 1.24 & 2.13 & 6.73 \\
\hline & $R-f$ & 4.72 & 2.35 & 7.06 & 0.98 & 2.01 & 7.16 \\
\hline \multirow{2}{*}{ Tartar } & I & 10.04 & 3.99 & 14.02 & 2.08 & 2.52 & 6.74 \\
\hline & R-f & 4.57 & 1.83 & 6.40 & 1.09 & 2.50 & 5.87 \\
\hline \multirow{2}{*}{ Sharg } & I & 5.16 & 1.93 & 7.08 & 1.46 & 2.67 & 4.84 \\
\hline & $R-f$ & 3.97 & 1.47 & 5.44 & 1.36 & 2.69 & 4.01 \\
\hline \multirow{2}{*}{ Gyrmyzybugda } & I & 5.84 & 2.07 & 7.91 & 1.47 & 2.81 & 5.38 \\
\hline & $R-f$ & 5.04 & 1.79 & 6.83 & 1.49 & 2.81 & 4.57 \\
\hline \multicolumn{8}{|c|}{ Triticum aestivum L. } \\
\hline \multirow{2}{*}{ Nurlu 99} & I & 6.06 & 2.42 & 8.48 & 1.45 & 2.51 & 5.83 \\
\hline & $R-f$ & 3.12 & 1.21 & 4.33 & 1.03 & 2.59 & 4.22 \\
\hline \multirow{2}{*}{ Gobustan } & I & 7.82 & 3.04 & 10.85 & 1.65 & 2.57 & 6.57 \\
\hline & $R-f$ & 3.23 & 1.35 & 4.58 & 0.82 & 2.40 & 5.56 \\
\hline \multirow{2}{*}{ Akinchi 84} & I & 7.98 & 3.12 & 11.10 & 1.87 & 2.55 & 5.92 \\
\hline & $R-f$ & 4.77 & 1.90 & 6.67 & 1.43 & 2.51 & 4.67 \\
\hline \multirow{2}{*}{ Giymatli 2/17 } & I & 6.63 & 2.87 & 9.50 & 1.37 & 2.31 & 6.93 \\
\hline & $R-f$ & 4.97 & 1.78 & 6.75 & 1.18 & 2.78 & 5.74 \\
\hline \multirow{2}{*}{ Gyrmyzygul 1} & I & 8.02 & 3.10 & 11.12 & 1.70 & 2.59 & 6.54 \\
\hline & R-f & 5.99 & 2.32 & 8.31 & 1.33 & 2.59 & 6.24 \\
\hline \multirow{2}{*}{ Azamatli95 } & I & 5.88 & 2.37 & 8.25 & 1.29 & 2.48 & 6.39 \\
\hline & R-f & 5.17 & 2.17 & 7.34 & 1.34 & 2.39 & 5.48 \\
\hline \multirow{2}{*}{ Tale38 } & I & 7.52 & 3.01 & 10.53 & 1.58 & 2.50 & 6.65 \\
\hline & $R-f$ & 5.86 & 2.26 & 8.12 & 1.44 & 2.59 & 5.62 \\
\hline \multirow{2}{*}{ Ruzi 84} & I & 5.92 & 2.23 & 8.15 & 1.6 & 2.66 & 5.09 \\
\hline & $R-f$ & 4.23 & 1.72 & 5.95 & 1.25 & 2.47 & 4.75 \\
\hline \multirow{2}{*}{ Pirshahin 1} & I & 5.31 & 2.56 & 7.87 & 1.21 & 2.08 & 6.50 \\
\hline & $R-f$ & 3.58 & 1.49 & 5.07 & 1.31 & 2.41 & 4.48 \\
\hline \multirow{2}{*}{$\begin{array}{c}\text { 12 } \\
\text { №97 } \\
\text { №wWON }\end{array}$} & I & 6.48 & 2.86 & 9.34 & 1.40 & 2.27 & 6.68 \\
\hline & $R-f$ & 5.25 & 2.24 & 7.49 & 1.41 & 2.34 & 5.30 \\
\hline \multirow{2}{*}{ Gunashli } & I & 6.46 & 2.44 & 8.90 & 1.59 & 2.64 & 5.60 \\
\hline & $R-f$ & 3.13 & 1.16 & 4.28 & 1.25 & 2.70 & 3.42 \\
\hline $4^{\text {th }}$ FEFWSN & I & 6.97 & 2.94 & 9.90 & 1.61 & 2.37 & 6.16 \\
\hline №50 & $R-f$ & 5.42 & 2.18 & 7.60 & 1.41 & 2.48 & 5.40 \\
\hline Dand & I & 7.34 & 2.77 & 10.11 & 1.73 & 2.65 & 5.83 \\
\hline Daguasit & R-f & 6.35 & 2.38 & 8.73 & 1.87 & 2.66 & 4.66 \\
\hline & I & 7.20 & 2.73 & 9.93 & 1.71 & 2.64 & 5.82 \\
\hline Saratovskaya 29 & R-f & 6.79 & 2.59 & 9.38 & 1.65 & 2.62 & 5.67 \\
\hline
\end{tabular}




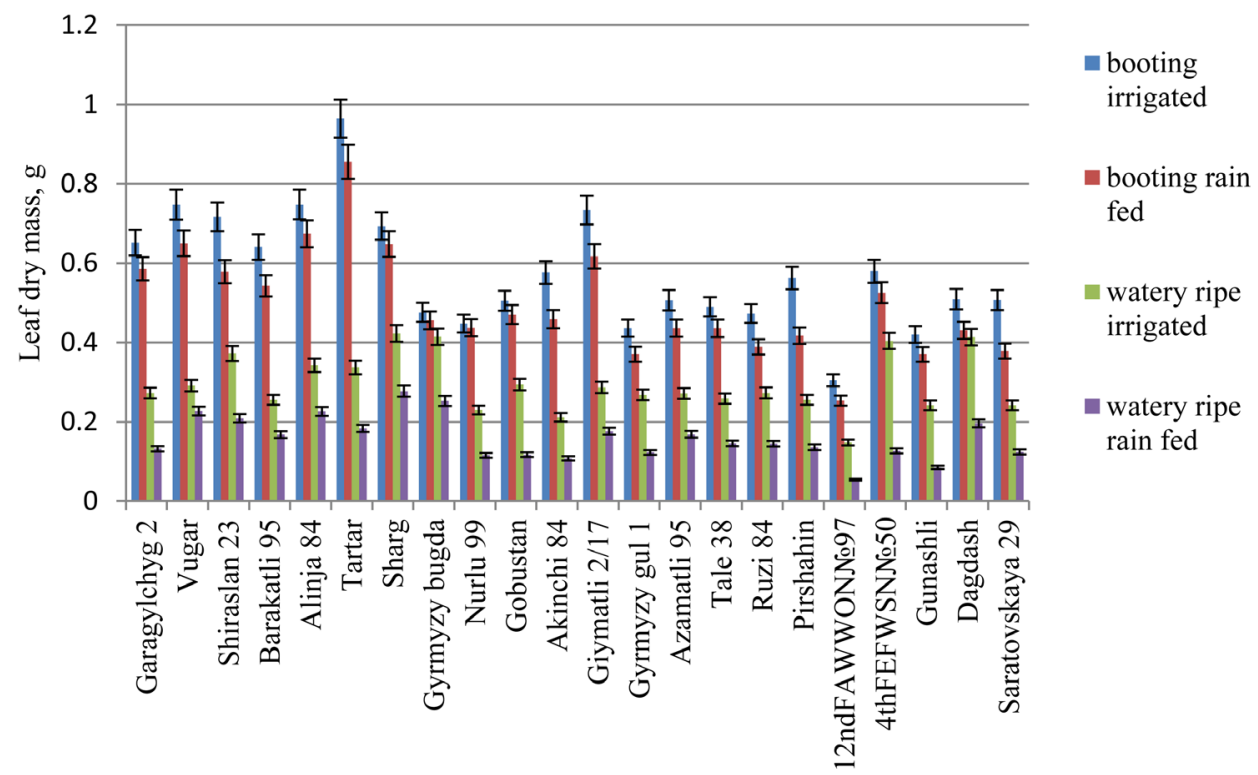

Figure 7. Effect of water stress on leaf dry mass per stem. Data are mean \pm SE from five replication.

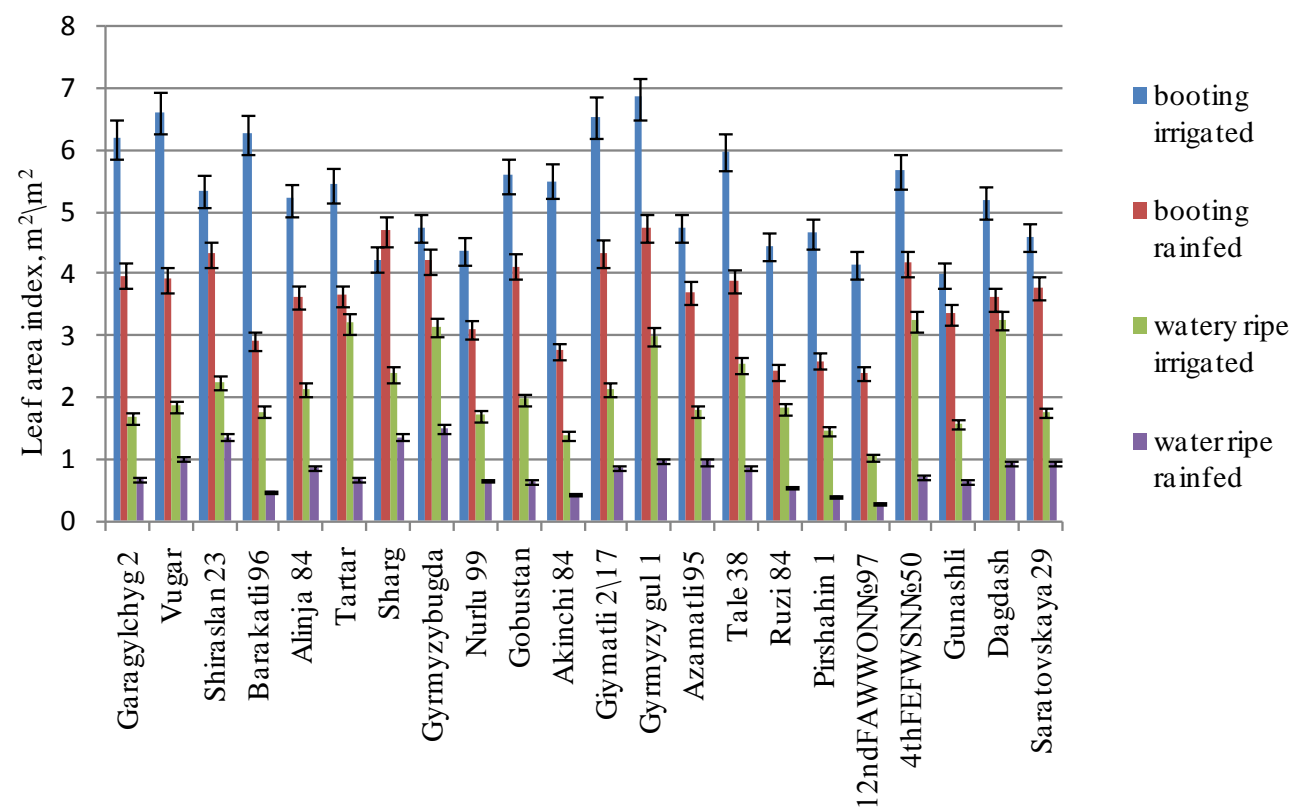

Figure 8. Effect of water stress on LAI. Data are mean \pm SE from three replication.

\subsection{Effect of Water Stress on Mesophyll Conductance $\left(g_{m}\right)$, Water Use Efficiency (WUE), Leaf Specific Mass (LSM)}

The $g_{m}$ calculated from $\mathrm{P}_{\mathrm{n}} / \mathrm{C}_{\mathrm{i}}$ ratio, WUE calculated from $\mathrm{P}_{\mathrm{n}} / \mathrm{E}$ ratio, and LSM calculated from the ratio of leaf dry mass to LA (Table 3). Lack of water in the soil led to a decrease in $g_{m}$, also in WUE. It was revealed an increase of LSM under water stress. The $g_{m}$ was higher in genotypes Tartar, Sharg, Giymatli 2/17, Gyrmyzy gul1, Tale 38, Ruzi 84, Pirshahin 1, Gunashli, Dagdash during booting stage, remained relatively higher in genotypes Shiraslan 23, Gyrmyzybugda, Gyrmyzy gul1, Pirshahin 1, Giymatli 2/17 and $4^{\text {th }}$ FEFWSN№50. Higher decrease of $g_{m}$ during both stages was observed in genotypes Alinja 84, Barakatli 95, Vugar, Nurlu 99, Akinchi 84, Saratovskaya 29. In some genotypes during booting stage water stress resulted in an increase of WUE. However during watery ripe stage the WUE decreased strongly due to deep reduction of $\mathrm{P}_{\mathrm{n}}$ than E. In terms of LSM no 
Table 3. Mesophyll conductance $\left(\mathrm{g}_{\mathrm{m}}\right)$, water use efficiency (WUE), leaf specific mass (LSM) of wheat genotypes.

\begin{tabular}{|c|c|c|c|c|c|c|c|c|c|c|c|c|}
\hline \multirow[b]{2}{*}{ Genotypes } & \multicolumn{4}{|c|}{$\mathrm{g}_{\mathrm{m}} \mathrm{mol} \mathrm{CO} \mathrm{Cm}^{-2} \cdot \mathrm{s}^{-1}$} & \multicolumn{4}{|c|}{ WUE. $\mu \mathrm{mol} \mathrm{CO} \mathrm{Cmol}^{-1} \mathrm{H}_{2} \mathrm{O}$} & \multicolumn{4}{|c|}{ LSM. $\mathbf{m g} \cdot \mathbf{m m}^{-2}$} \\
\hline & 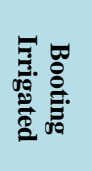 & 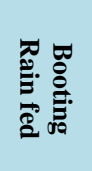 & 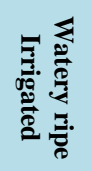 & . & 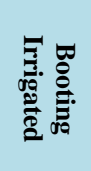 & 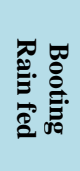 & の & 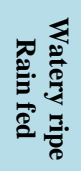 & 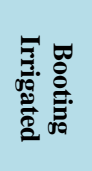 & 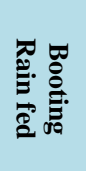 & 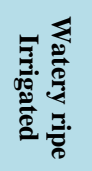 & 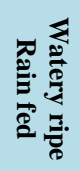 \\
\hline \multicolumn{13}{|c|}{ Triticum durum (Desf.) } \\
\hline Garagylchyg 2 & 0.034 & 0.019 & 0.019 & 0.010 & 5.27 & 5.18 & 1.54 & 1.10 & 0.044 & 0.052 & 0.068 & 0.069 \\
\hline Vugar & 0.021 & 0.013 & 0.020 & 0.008 & 4.29 & 5.07 & 1.74 & 0.83 & 0.042 & 0.045 & 0.059 & 0.061 \\
\hline Shiraslan 23 & 0.042 & 0.025 & 0.017 & 0.012 & 6.68 & 5.70 & 1.81 & 1.35 & 0.043 & 0.044 & 0.053 & 0.051 \\
\hline Barakatli 95 & 0.037 & 0.020 & 0.014 & 0.004 & 5.08 & 4.39 & 1.66 & 0.61 & 0.039 & 0.039 & 0.055 & 0.073 \\
\hline Alinja 84 & 0.041 & 0.026 & 0.023 & 0.007 & 5.07 & 4.80 & 2.38 & 1.09 & 0.043 & 0.047 & 0.048 & 0.066 \\
\hline Tartar & 0.043 & 0.036 & 0.031 & 0.009 & 5.85 & 6.22 & 2.03 & 1.16 & 0.055 & 0.055 & 0.032 & 0.064 \\
\hline Sharg & 0.068 & 0.038 & 0.011 & 0.010 & 3.26 & 2.99 & 1.02 & 1.05 & 0.050 & 0.049 & 0.054 & 0.073 \\
\hline Gyrmyzybugda & 0.043 & 0.027 & 0.021 & 0.020 & 3.06 & 2.30 & 1.61 & 1.60 & 0.043 & 0.042 & 0.057 & 0.066 \\
\hline \multicolumn{13}{|c|}{ Triticum aestivum L. } \\
\hline Nurlu 99 & 0.060 & 0.026 & 0.009 & 0.003 & 5.10 & 4.47 & 1.29 & 0.57 & 0.044 & 0.048 & 0.057 & 0.061 \\
\hline Gobustan & 0.061 & 0.026 & 0.012 & 0.010 & 4.54 & 3.86 & 1.58 & 1.09 & 0.041 & 0.049 & 0.068 & 0.078 \\
\hline Akinchi 84 & 0.062 & 0.021 & 0.022 & 0.008 & 4.90 & 3.71 & 1.74 & 1.03 & 0.044 & 0.051 & 0.064 & 0.078 \\
\hline Giymatli 2/17 & 0.063 & 0.044 & 0.035 & 0.013 & 5.28 & 5.11 & 2.05 & 1.07 & 0.048 & 0.042 & 0.058 & 0.067 \\
\hline Gyrmyzy gul1 & 0.047 & 0.035 & 0.025 & 0.018 & 4.32 & 5.67 & 1.71 & 1.57 & 0.040 & 0.043 & 0.056 & 0.069 \\
\hline Azamatli 95 & 0.056 & 0.030 & 0.016 & 0.013 & 4.45 & 4.00 & 1.16 & 1.20 & 0.047 & 0.050 & 0.068 & 0.075 \\
\hline Tale 38 & 0.046 & 0.043 & 0.029 & 0.008 & 4.40 & 4.28 & 1.65 & 0.91 & 0.044 & 0.046 & 0.054 & 0.069 \\
\hline Ruzi 84 & 0.051 & 0.034 & 0.029 & 0.006 & 4.10 & 4.31 & 1.96 & 0.85 & 0.049 & 0.060 & 0.069 & 0.099 \\
\hline Pirshahin 1 & 0.073 & 0.059 & 0.032 & 0.017 & 4.56 & 4.69 & 2.20 & 1.34 & 0.053 & 0.054 & 0.077 & 0.113 \\
\hline $12^{\text {nd }}$ FAWWON97 & 0.031 & 0.024 & 0.018 & 0.007 & 3.51 & 3.81 & 1.39 & 0.58 & 0.048 & 0.060 & 0.093 & 0.100 \\
\hline Gunashli & 0.038 & 0.031 & 0.016 & 0.004 & 3.62 & 4.18 & 1.10 & 0.40 & 0.046 & 0.048 & 0.056 & 0.067 \\
\hline $4^{\text {th }}$ FEFEWSN50 & 0.057 & 0.024 & 0.025 & 0.013 & 3.21 & 2.60 & 1.21 & 0.79 & 0.049 & 0.053 & 0.071 & 0.064 \\
\hline Dagdash & 0.040 & 0.032 & 0.021 & 0.008 & 3.18 & 2.86 & 1.25 & 0.72 & 0.043 & 0.043 & 0.057 & 0.075 \\
\hline Saratovskaya 29 & 0.037 & 0.013 & 0.017 & 0.005 & 3.19 & 1.80 & 1.24 & 0.56 & 0.052 & 0.048 & 0.065 & 0.063 \\
\hline
\end{tabular}

strict difference between irrigated and rain-fed plants observed during booting stage. In some genotypes (Barakatli 95, Alinja 84, Tartar, Ruzi 84, Pirshahin1) there were big differences in LSM of irrigated and rain-fed plants during watery ripe stage.

\subsection{Effect of Drought Stress on Grain Yield}

Most genotypes of bread wheat formed more yield than genotypes of durum wheat under irrigated condition (Figure 9). Soil water deficit led to a strong decrease in grain yield of genotypes Vugar, Barakatli 95, Alinja 84, Tartar, Tale 38, Akinchi 84, Giymatli 2/17, Gyrmyzy gul 1, Gobustan, Azamatli 95, Ruzi 84, Pirshahin 1, less reduction was observed in genotypes Shiraslan 23, Sharg, Gyrmyzybugda, Nurlu 99, 12 ${ }^{\text {nd }}$ FAWWON№97, $4^{\text {th }}$ FEFWSN№50, Saratovskaya 29. 


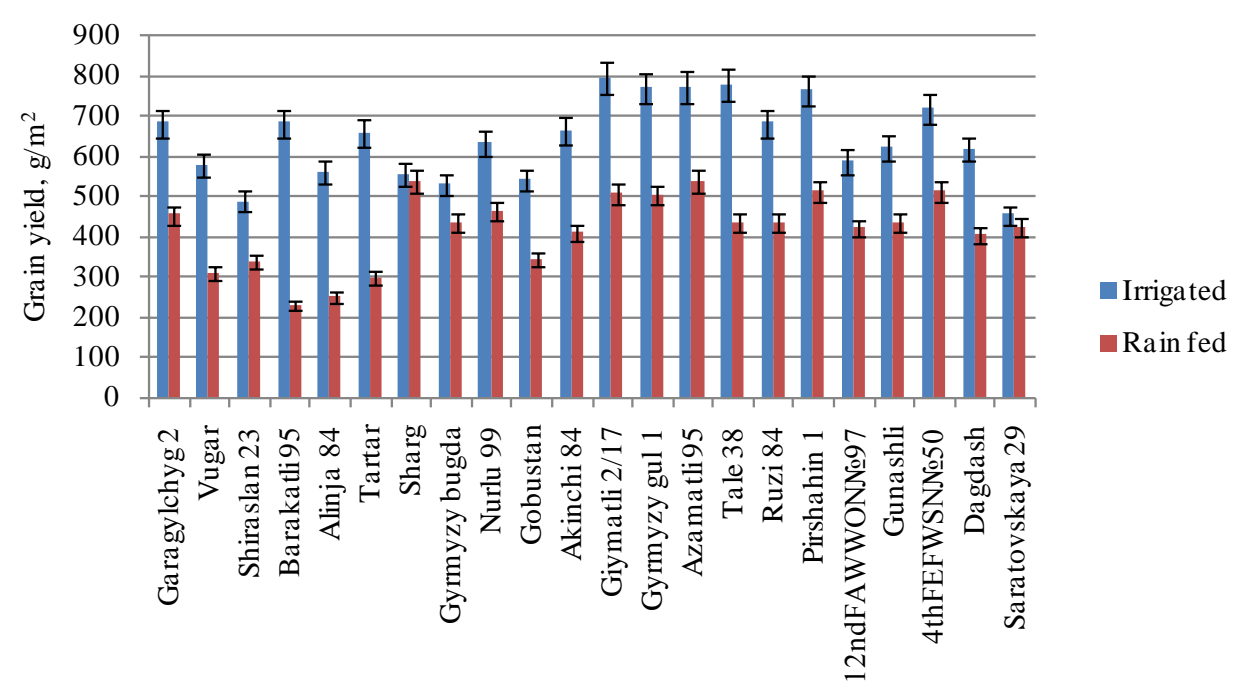

Figure 9. Effect of water stress on grain yield. Data are mean \pm SE from three replication.

\section{Discussion}

Water shortage led to a decrease in the $P_{n}, g_{s}$ and E, $g_{m}$, WUE, an increase in $C_{i}$. Despite the fact that the $g_{s}$, and E were relatively high during the watery ripe stage the $P_{n}$ strongly suppressed. This fact indicates predominance of nonstomatal factors in regulation of $P_{n}$. Our previous results showed that $g_{m}$ is a dominant in regulation of $P_{n}$ [15]. We found an increase in the ratio of $C_{i} / C_{a}$ under rain-fed conditions $\left(C_{a}\right.$-ambient $\mathrm{CO}_{2}$ concentration). The $\mathrm{Chl}(\mathrm{a}+\mathrm{b}), \operatorname{Car}(\mathrm{x}+\mathrm{c})$ content of wheat genotypes decreased under rain-fed conditions. We detected decrease of $\mathrm{Chl} \mathrm{a/b}$ ratio, no changes or an increase of Chla/b ratio in some of genotypes. There were reported a slight rise in Chla/b ratio in drought tolerant wheat cultivars and a significant decrease in the susceptible ones under water deficit conditions (PEG-6000 at -0.6MPa) [16]. In addition, a decline of Chla/b ratios has been associated with lower drought resistance, while a decline of Car/Chl suggested that the development of light-harvesting complex and the dissipation of thermal energy might be strongly affected by water deficit since Cars of chloroplast are major components of antenna system [17] [18]. The RWC of studied durum and bread wheat genotypes less affected by water stress than gas exchange parameters and pigments content. The $\mathrm{g}_{\mathrm{s}}$ is more closely linked to soil moisture content than to leaf water status [19]. The RWC of three sunflower cultivars reduced by $3 \%$ under drought stress as an average of all genotypes [20]. It is reported that high RWC is a resistant mechanism to drought, and that high RWC is the result of more osmotic regulation or less elasticity of tissue cell wall [21]. The genotypes of durum wheat formed more LA and dry mass than the genotypes of bread wheat. However the bread wheat genotypes formed more tillers than the durum wheat genotypes (data not shown). The LSM of most wheat genotypes was increased under rain fed condition. Such an increase in the LSM is probably adaptive response to drought and is due to the relatively greater reduction in LA than the dry mass. A large difference in LSM of some wheat genotypes Barakatli 95, Alinja 84, Tartar, Pirshahin 1, Ruzi 84 indicate that this genotypes could not send sufficient assimilates to spike under water stress. Drought tolerant wheat genotypes showed escape mechanisms consisting of good winter growth (early vigour), hence high pre-anthesis values of LA and LA duration, high translocation of pre-anthesis assimilate to the ear [22] and hence poor dry matter content per unit leaf area (low specific leaf mass, small leaf thickness) after anthesis [23]. Some of our studied genotypes (Nurlu 99, Gobustan, Azamatli 95, Akinchi 84) have earlier heading time that allows drought escape and have more time interval to send assimilates to spike. Drought caused reduction of yield of this genotype was not severe. Some genotypes with middle or late heading time (Garagylchyg 2, Alinja 84, Giymatli 2/17, Gyrmyzygul 1, Tale 38, Ruzi 84, Pirshahin 1, $4^{\text {th }}$ FEFWSN50) showed high $\mathrm{P}_{\mathrm{n}}$ under irrigated condition, but this parameter severely reduced under water stress conditions. Our results showed that the genotype $12^{\text {nd }}$ FAWWON№97 had the smallest LA, lowest $\mathrm{P}_{\mathrm{n}}$ and $\mathrm{E}$ but had a high LSM and reduction in yield was low. Despite the fact that drought stress led to considerable reduction in grain yield of genotype Vugar, decrease in LA, dry mass, Chl(a+b) content, RWC was slightly. Water deficit more affected on grain yield of durum wheat genotypes than bread wheat. Drought caused less reduction in grain yield of genotypes Shiraslan 23, Sharg, Gyrmyzybugda, Nurlu 99, 
$12^{\text {nd }}$ FAWWON№97, $4^{\text {th }}$ FEFWSN№50, Saratovskaya 29. We can consider these genotypes as drought resistant. Summarizing the results of the study, we can assume that the photosynthetic apparatus of genotypes Vugar, Shiraslan 23, Sharg, Gyrmyzybugda, Gyrmyzy gul1, Giymatli 2/17, $4^{\text {th }}$ FEFWSN50, $12^{\text {nd }}$ FAWWON97, Tale38, Pirshahin 1, Dagdash, Saratovskaya 29 was more resistant to drought stress.

\section{Conclusion}

Water scarcity in the field led to adaptive changes in physiological traits of durum wheat and bread wheat genotypes. Reduction of $g_{s}$ results in a decrease of $P_{n}$ and $E$, an increase of $C_{i}$. Close strict decrease in the RWC and $\mathrm{Chl}(\mathrm{a}+\mathrm{b})$ content was observed in some genotypes under the influence of drought. Water shortage led to reduction of LA, dry mass and LAI which is strongly expressed during watery ripe stage. Different wheat genotypes have some advantages in agronomic, morphophysiological parameters, which can reduce the damage caused by water stress. Physiological studies on field grown wheat genotypes can be useful for identification of drought resistant genotypes.

\section{References}

[1] Ludlow, M.M. and Muchow, R.C. (1990) A Critical Evaluation of Traits for Improving Crop Yields in Water-Limited Environments. Advances in Agronomy, 43, 107-153. http://dx.doi.org/10.1016/S0065-2113(08)60477-0

[2] Bogale, A., Tesfaye, K. and Geleto, T. (2011) Morphological and Physiological Attributes Associated to Drought Tolerance of Ethiopian Durum Wheat Genotypes under Water Deficit Condition. Journal of Biodiversity and Environmental Sciences, 1, 22-36.

[3] Rajaram, S. (2001) Prospects and Promise of Wheat Breeding in the $21^{\text {st }}$ Century. Euphytica, 119, 3-15. http://dx.doi.org/10.1023/A:1017538304429

[4] Arora, A., Sairam, R.K. and Srivastava, G.C. (2002) Oxidative Stress and Oxidative System in Plants. Current Science, 82, 1227-1238.

[5] Tang, A.C., Kawamitsa, Y., Kanechi, M. and Boyer, J.S. (2002) Photosynthesis at Low Water Potentials in Leaf Discs Lacking Epidermis. Annals of Botany, 89, 861-870. http://dx.doi.org/10.1093/aob/mcf081

[6] Pinheiro, C. and Chaves, M.M. (2011) Photosynthesis and Drought: Can We Make Metabolic Connections from Available Data? Journal of Experimental Botany, 62, 869-882. http://dx.doi.org/10.1093/jxb/erq340

[7] Akram, M.S. and Ashraf, M. (2011) Exogenous Application of Potassium Dehydrogen Phosphate Can Alleviate the Adverse Effects of Salt Stress on Sunflower (Helianthus annuus L.). Journal of Plant Nutrition, 34, 1041-1057. http://dx.doi.org/10.1080/01904167.2011.555585

[8] Perveen, S., Shahbaz, M. and Ashraf, M. (2010) Regulation in Gas Exchange and Quantum Yield of Photosystem II (PSII) in Salt-Stressed and Non-Stressed Wheat Plants Raised from Seed Treated with Triacontanol. Pakistan Journal of Botany, 42, 3073-3081.

[9] Kaewsuksaeng, S. (2011) Chlorophyll Degradation in Horticultural Crops. Walailak Journal of Science and Technology, 8, 9-19.

[10] Akram, M. (2011) Growth and Yield Components of Wheat under Water Stress of Different Growth Stages. Bangladesh Journal of Agricultural Research, 36, 455-468. http://dx.doi.org/10.3329/bjar.v36i3.9264

[11] Khakwani, A.A., Dennet, M.D. and Munir, M. (2011) Drought Tolerance Screening of Wheat Varieties by Inducing Water Stress Conditions. Songklanakarin Journal of Science and Technology, 33, 135-142.

[12] Lichtenthaler, H.K. (1987) Chlorophylls and Carotenoids: Pigments of Photosynthetic Biomembranes. Methods in Enzymology, 148, 350-382. http://dx.doi.org/10.1016/0076-6879(87)48036-1

[13] Richards, A. (2000) Selectable Traits to Increase Crop Photosynthesis and Yield of Grain Crops. Journal of Experimental Botany, 51, 447-458. http://dx.doi.org/10.1093/jexbot/51.suppl_1.447

[14] Rucker, K.S., Kvien, C.K., Holbrook, C.C. and Hook, J.E. (1995) Identification of Peanut Genotypes with Improved Drought Avoidance Traits. Peanut Science, 22, 14-18. http://dx.doi.org/10.3146/pnut.22.1.0003

[15] Allahverdiyev, T.I., Talai, J.M., Huseynova, I.M. and Aliyev, J.A. (2015) Effect of Drought Stress on Some Physiological Parameters, Yield, Yield Components of Durum (Triticum durum Desf.) and Bread (Triticum aestivum L.) Wheat Genotypes. Ekin Journal of Crop Breeding and Genetics, 1-1, 50-62.

[16] Ashraf, M.Y., Azmi, A.R., Khan, A.H. and Ala, S.A (1994) Effect of Water Stress on Total Phenol, Peroxidase Activity and Chlorophyll Contents in Wheat (Triticum aestivum L.). Acta Physiologiae Plantarum, 16, 185-191.

[17] Zhang, Y.X., Wu,, J.C., Cao F.L. and Zhang, Y.P. (2010) Effects of Water Stress on Photosynthetic Activity, Dry Mass Partitioning and Some Associated Metabolic Changes in Four Provenances of Neem (Azadirachta indica A. Juss). 
Photosynthetica, 48, 361-369. http://dx.doi.org/10.1007/s11099-010-0047-y

[18] Jeon, M.W., Ali, M.B., Hahn, E.J. and Paek, K.Y. (2006) Photosynthetic Pigments, Morphology and Leaf Gas Exchange during ex Vitro Acclimatization of Micropropagated CAM Doritaenopsis Plantlets under Relative Humidity and Air Temperature. Environmental and Experimental Botany, 55, 183-194.

http://dx.doi.org/10.1016/j.envexpbot.2004.10.014

[19] Davies, W.J. and Zhang, J.H. (1991) Root Signals and the Regulation of Growth and Development of Plants in Drying Soil. Annual Review of Plant Physiology and Plant Molecular Biology, 42, 55-76. http://dx.doi.org/10.1146/annurev.pp.42.060191.000415

[20] Canavar, Ö., Götz, K.P., Ellmer, F., Chmielewski, F.M. and Kaynak, M.A. (2014) Determination of the Relationship between Water Use Efficiency, Carbon Isotope Discrimination and Proline in Sunflower Genotypes under Drought Stress. Australian Journal of Crop Science, 8, 232-242.

[21] Ritchie, S.W., Nguyan, H.T. and Holaday, A.S. (1990) Leaf Water Content and Gas Exchange Parameters of Two Wheat Genotypes Differing in Drought Resistance. Crop Science, 30, 105-111. http://dx.doi.org/10.2135/cropsci1990.0011183X003000010025x

[22] Wittmer, G., Iannucci, A., De Santis, G., Balzelli, G., De Stefanis, E., Brando, A., Ciocca, L. and Rascio, A. (1982) Adattamento e fattorifisiologicilimitanti la produttivita del frumentoduro. Rivisita di Agronomia, 2, 61-70.

[23] Cedola, M.C., Iannucci, A., Scalfati, G., Soprano, M. and Rascio, A. (1994) Leaf Morpho-Physiological Parameters as Screening Techniques for Drought Stress Tolerance in Triticum durum Desf. Journal of Genetics and Breeding, XLVIII, 229-235. 